\title{
The Incidence and Prognosis of ICU Delirium: A Retrospective Study from a Single Center
}

\author{
Qing Feng ${ }^{1,2}$, Yuhang $\mathrm{Ai}^{1}$, Meilin $\mathrm{Ai}^{1}$, Li Huang ${ }^{1}$, Qianyi Peng ${ }^{1}$, Lina Zhang ${ }^{1, *}$ \\ ${ }^{1}$ Department of Intensive Care Unit, Xiangya Hospital of Central South University, Changsha 410008, China \\ ${ }^{2}$ Department of Intensive Care Unit, Shenzhen Hospital of Peking University, Shenzhen 518036, China
}

\section{ARTICLE INFO}

\section{Article History}

Received 09 December 2020

Accepted 05 February 2021

Keywords

Delirium

ICU

sleep quality

28-day mortality

length of ICU stay

\begin{abstract}
Purpose: Delirium is a frequent occurrence amongst Intensive Care Unit (ICU) patients, but studies on its causes are sparse and its risk factors vary from disease to disease. Here, in this study, an attempt was made to investigate the factors associated with the prognosis of delirium in the ICU.

Methods: An observed study was performed of consecutive patients from September 2016 to November 2016 who were admitted to the ICU. The patients were screened for delirium by using the diagnostic tools of the Richmond Agitation Sedation Scale score and the Confusion Assessment Method ICU. Patients were divided into subgroups according to heart/vascular, cerebral, pulmonary, maxillofacial/limbs, obstetric, urinary and spinal disease.

Results: A total of 406 patients of which 186 developed delirium (45.8\%). The main causes of delirium were different. The only independent factor for delirium in heart and vascular subgroups was the quality of sleep (Odds Ratio (OR) $=0.236, p<$ $0.001[0.111-0.500])$. For those with intestinal disease, the risk factors included age $(\mathrm{OR}=2.514, p=0.002[1.397-4.524])$, use of vasoactive therapies $(\mathrm{OR}=13.799, p=0.002[2.669-71.361])$ and the quality of sleep $(\mathrm{OR}=0.114, p<0.001[0.036-0.366])$. Older age $(\mathrm{OR}=1.100, p=0.022$ [1.014-1.194]), higher acute physiology, age and chronic health evaluation II scales (OR = $1.255, p<0.001[1.112-1.417])$ and the quality of sleep $(\mathrm{OR}=0.090, p=0.034[0.010-0.829])$ were noted as risk factors for septic shock patients. Delirium led to extended ICU-stays $(p<0.001)$, and only the subgroups of septic shock patients showed a difference in 28-day mortality rates $(p=0.006)$.
\end{abstract}

Conclusion: The incidence of delirium and its associated risk factors varied according to disease type. In the study cohort, the highest 28-day mortality was recorded for the patients admitted for septic shock who had developed delirium.

(c) 2021 First Affiliated Hospital of Zhengzhou University. Publishing services by Atlantis Press International B.V. This is an open access article distributed under the CC BY-NC 4.0 license (http://creativecommons.org/licenses/by-nc/4.0/).

\section{INTRODUCTION}

Intensive Care Units (ICUs) are specialist wards providing intensive care to critically ill patients or to those in an unstable condition. ICUs are typically multidisciplinary, admitting patients with a range of pathologies that require intensive or high dependency care due to disease severity or unstable disease presentation [1]. Given the severity of the condition of the ICU patients, delirium is a common occurrence, with incidence rates ranging from $11 \%$ to $80 \%$ reported in previous studies [1-3]. Delirium is characterized by transient states of cognition/consciousness [4] that can lead to extended ICU stays, loss of cognitive function, mortality or a heightened risk of death after 6 months. The disorder, therefore, represents a burden to patients, relatives, and health care systems [5-7].

The treatment of delirium in the ICU is dependent on its early diagnosis, the alleviation of modifiable risk factors, and the treatment of predisposing factors [8]. Previous [9] studies identified age,

"Corresponding author.Email: zln7095@csu.edu.cn

Peer review under responsibility of the First Affiliated Hospital of Zhengzhou University

Data availability statement: The datasets used and/or analyzed during the current study are available from the corresponding author [LNZ], upon reasonable request.
Complexity Prediction Instrument (COMPRI) scores, and psychosocial vulnerabilities as risk factors. Tse et al. [10] performed a retrospective analysis that identified a history of delirium, in different patient populations with independent risk factors such as Cardiopulmonary Bypass Graft in patients aged $\geq 64$ years, stroke patients, those experiencing a transient ischemic attack, depression, a loss of cognitive function, and beta-blocker usage prior to surgery. Wang et al. [11] further showed that age ( $>70$ years), anesthesia, hypercapnia during operation, surgery lasting over $3 \mathrm{~h}$, hypotension, and sleep disorders were associated with delirium in geriatrics undergoing orthopedic surgery. The occurrence of delirium varied according to disease type.

A diagnosis for delirium can be made based on medical history, the assessment of mental status and the identification of possible contributing factors. Typical examinations include mental status examinations that include assays of awareness, attention and thinking, either informally or through conversation; assessments of mental state, confusion, perception and memory; physical and neurological exams and the analysis of underlying disease. The assessment of delirium in the ICU setting includes the Confusion Assessment Method for the ICU (CAM-ICU) [12] and the Intensive Care Delirium Screening Checklist $[13,14]$. The CAM-ICU is verified as 
an accurate predictor of delirium occurrence in critically ill patients [15]. In the present study, we investigated the risks of delirium in ICU patients to identify specific characteristics as prognostic indicators. We focused on subjects with a range of disease types to improve disease prognosis and identification. Since the causes and occurrence of delirium in the ICU vary according to disease-type, our study aimed to identify novel independent risk factors in the patients admitted to ICU. The results of this study would offer new insights for patient management and treatment in the ICU setting.

\section{MATERIALS AND METHODS}

\subsection{Patient Setting}

The study was performed in a large mixed medical ICU from September 2016 to November 2016 in Changsha, China. The ICU was isolated from the rest of the hospital and was managed by accredited intensivists responsible for patient care. Ward rounds were performed twice per day and treatment plans were regularly reviewed. The lights of the ICU were dimmed to promote sleep.

\subsection{Study Population}

The inclusion criteria in this study were therefore as follows: age $>18$ years; ICU treated for $>24 \mathrm{~h}$. Informed consent was provided by the patient or next of kin.

Exclusion criteria: known sleep disorders, drug-treated mental illness, psychological issues, cognitive disorders, dementia or central neurological impairment diagnosed by radiological scans; and Richmond Agitation-Sedation Scale (RASS) scores $\geq 4$ during the ICU stay.

\subsection{Data Collection}

Patient data were collected on weekdays and included characteristics such as gender, age, alcohol and nicotine intake, diabetes, hypertension and vascular disease. The cut-off time was 28 days.

\subsection{Delirium Measurements}

The symptoms of delirium were assessed twice daily by two ICU clinicians using the CAM-ICU scales. Delirium was defined as: (1) acute changes or fluctuations in mental state; (2) a loss of attention; (3) changes in consciousness; and (4) unordered thoughts. Delirium was present for values of: $1+2+3$ or $1+2+4$ [16]. Patients with delirium were considered positive when presenting at least one positive CAM-ICU during the ICU stay.

\subsection{Assessment of Diseases Types}

Patients were divided into subgroup according to heart/vascular disease, cerebral disorders, pulmonary disease, disease of the maxillofacial/limbs, gynecologic disorders, obstetric disease, urinary and spinal disease. Sepsis 3.0 was used to diagnose septic shock [17].

\subsection{Statistics}

Data were represented as mean $\pm \mathrm{SD}$, and were analyzed using SPSS 18.0 software (Armonk, New York). Differences amongst the groups were compared using a $t$-test for normally distributed data, and a Wilcoxon rank-sum test for non-normal data, represented by $M(\mathrm{P} 25, \mathrm{P} 75) . \chi^{2}$ tests were used to compare categorical variables (theoretical frequency $<5$ for continuous correction methods). For theoretical frequencies $<1$, exact probability methods were adopted. Kaplan-Meier (KM) curves were analyzed using log-rank tests. Survival analyses were performed via stepwise Cox regression models. $p<0.05$ and $p<0.01$ were deemed statistically significant.

\section{RESULTS}

This was an observational study of consecutive patients from September 2016 to November 2016 admitted to the ICU for surgery or treatment. In total, $42 / 459$ patients were excluded, with 11 not completing the delirium assessments. Therefore, 406 patients were enrolled following CAM-ICU evaluations. Of these patients, 107 had vascular and cardiac disease, 107 had intestinal disease, 41 had diseases of the pulmonary system, 37 suffered from septic shock, 34 had cerebral disease, 29 had maxillofacial/limbs, 21 had gynecologic disease, 17 had urinary disease, and 13 had diseases of the spine (Figure 1).

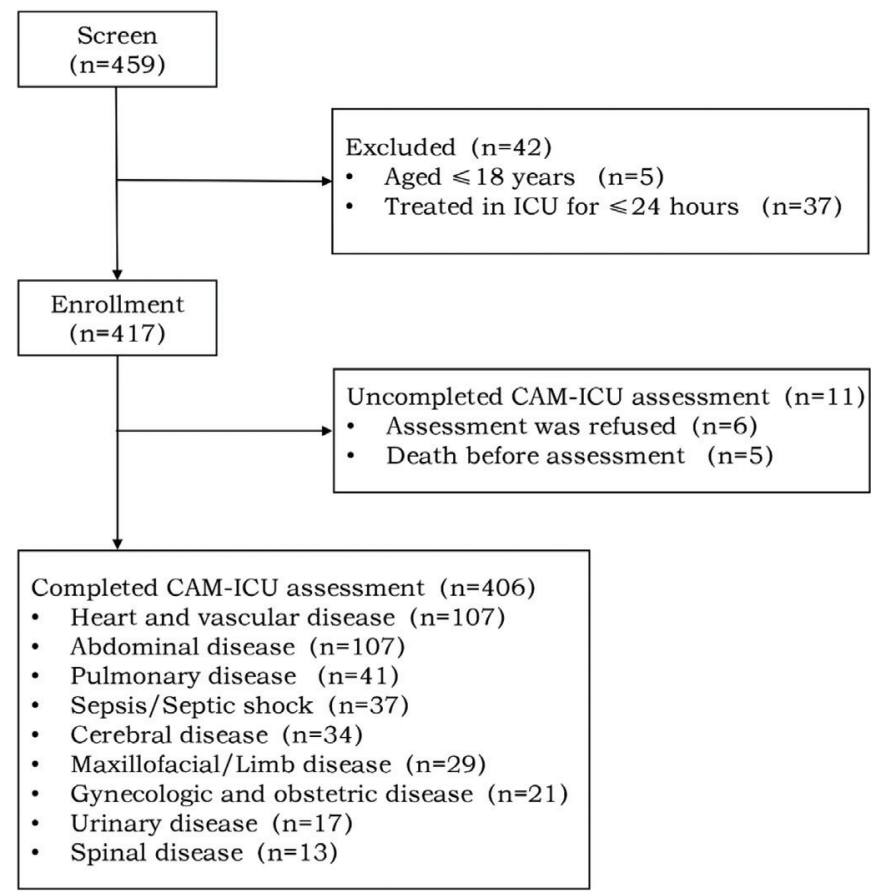

Figure 1 Flow diagram of the study protocol. A total of 406 patients were finally enrolled. Inclusion criteria: age $>18$ years; ICU treatment for $>24 \mathrm{~h}$. Informed consent provided by the patient or next of kin. Exclusion criteria: known sleep disorders, drug treated mental illness, psychological issues, cognitive disorders, dementia or central neurological impairment diagnosed by radiological scans; and RASS scores $\geq 3$ during the ICU stay. 


\subsection{Biochemical Indicators and Baseline Features}

Of the 406 included patients, 186 (45.8\%) suffered from delirium in the ICU. The patients with delirium were aged $60 \pm 14$ years $(n=186)$ in comparison to $50 \pm 16$ years for ICU patients without delirium $(n=220, p<0.001)$. Compared to those without delirium, sequential organ failure assessment $(8 \pm 5$ vs $5 \pm 4)$ and Acute Physiology, Age and Chronic Health Evaluation (APACHE II) $(14 \pm 6$ vs $9 \pm 5 ; p<0.001)$ scores were higher in delirium patients. The patients with delirium had a poor sleep quality $(p<0.001)$, hypertension (39\% vs $25 \%, p=0.003)$, were sedated (82\% vs $65 \% ; p<0.001)$, had high multiple organ dysfunction syndrome scores ( $40 \%$ vs $21 \%$; $p<0.001$ ) high levels of vasoactive drug administration ( $39 \%$ vs $29 \%$; $p=0.04)$. Longer ventilation times $(11 \mathrm{~h}[3,27]$ vs $5 \mathrm{~h}[1,10] ; p<0.001)$, longer duration of ICU stay $(5.5 \pm 5.4$ vs $3.0 \pm 2.5 ; p<0.001)$ and higher 28 -day mortality rates $(13 \%$ vs $4 \% ; p<0.001)$ were reported for those that developed delirium (Table 1 ).

In terms of biochemical indicators, the non-delirium group had higher levels of BUN $(8.5[5.4,12.7]$ vs $6.7[4.3,10.2], p<0.001)$ PCT $(2.0[0.2,12.1]$ vs $0.9[0.2,4.7], p=0.023)$, and $\operatorname{Cr}(110.0$ [83.0, 165.0] vs $92.0[72.0,123.5], p=0.027)$, whilst PLT levels declined $(146.5 \pm 73.2$ vs $162.7 \pm 87.9, p=0.047)$. No differences were observed between the other indicators (Table 1).

\subsection{Delirium According to Disease Types}

A total of 406 patients were studied of which 186 developed delirium (45.8\%). We found that the highest incidence of delirium was amongst the subgroup of cerebral disease (68\%), followed by pulmonary disorders (63\%), maxillofacial/limbs (52\%), septic shock $(49 \%)$, cardiac and vascular disease (44\%) and intestinal disease (42\%) (Figure 2).

Considering the occurrence of delirium as a dependent covariate, we used multiple logistic regression for data comparisons. Logistic regression variables were then introduced into the univariate analysis, including those with $p$-values $<0.05$. From these analyses, we observed that older patients were likely to develop delirium (Odds Ratio $(\mathrm{OR})=1.756, p=0.001[1.406-2.194])$ as were those with higher APACHE II scores (OR = 1.093, $p=0.001$ [1.039-1.150]). Patients with a longer duration of ventilation also showed increased susceptibility to the development of delirium $(\mathrm{OR}=1.022, p=0.012$ [1.005-1.040]). Sedated patients $(\mathrm{OR}=3.406, p<0.001$ [1.8676.212]) were prone to delirium, whist a good sleep quality was protective against the onset of delirium $(\mathrm{OR}=0.186, p<0.001$ [0.121-0.288]) (Table 2).

Regarding disease status, delirium development in cardiac patients was dependent on sleep levels $(\mathrm{OR}=0.236, p<0.001$ [0.111-0.500]). For ICU patients with intestinal disease, the use of vasoconstrictive agents $(\mathrm{OR}=13.799, p=0.002[2.669$ $71.361])$, older age $(\mathrm{OR}=2.514, p=0.002[1.397-4.524])$ and low levels of sleep $(\mathrm{OR}=0.114, p<0.001[0.036-0.366])$ were risk factors. For septic shock patients, higher APACHE II scores $(\mathrm{OR}=1.255, p<0.001[1.112-1.417])$, low sleep levels $(\mathrm{OR}=0.090$,
Table 1 Baseline characteristics stratified by delirium

\begin{tabular}{|c|c|c|c|}
\hline Variables & $\begin{array}{c}\text { Delirium } \\
(n=186) N(\%)\end{array}$ & $\begin{array}{l}\text { Non delirium } \\
(n=220) N(\%)\end{array}$ & $p$-value \\
\hline Age, mean $(S D)^{a}$, years & $60 \pm 14$ & $50 \pm 16$ & $<0.001$ \\
\hline Men & $101(54)$ & $135(61)$ & 0.151 \\
\hline \multicolumn{4}{|l|}{ Medical history } \\
\hline Hypertension & $73(39)$ & $56(25)$ & 0.003 \\
\hline Cardiac disease & $55(30)$ & $48(22)$ & 0.074 \\
\hline Diabetes & $21(11)$ & $18(8)$ & 0.29 \\
\hline Smoking history & $48(26)$ & $73(33)$ & 0.105 \\
\hline Drinking history & $24(13)$ & $28(13)$ & 0.958 \\
\hline \multicolumn{4}{|l|}{ Baseline features } \\
\hline $\begin{array}{l}\mathrm{APACHE}^{\mathrm{b}} \text { II score, } \\
\text { mean (SD) }\end{array}$ & $14 \pm 6$ & $9 \pm 5$ & $<0.001$ \\
\hline $\begin{array}{c}\text { SOFAc }^{c} \text { score } \\
\text { mean }(\mathrm{SD})\end{array}$ & $8 \pm 5$ & $5 \pm 4$ & $<0.001$ \\
\hline MODS & $75(40)$ & $46(21)$ & $<0.001$ \\
\hline $\begin{array}{l}\text { Mechanical } \\
\text { ventilation }\end{array}$ & $156(84)$ & $171(78)$ & 0.119 \\
\hline $\begin{array}{l}\text { Duration of } \\
\text { ventilation, } \\
\text { median (IQR), h }\end{array}$ & $11[3,27]$ & $5[1,10]$ & $<0.001$ \\
\hline Sedation & $152(82)$ & $143(65)$ & $<0.001$ \\
\hline Vasoactive drugs & $72(39)$ & $64(29)$ & 0.04 \\
\hline \multicolumn{3}{|c|}{ Sleep quality assessment (PSQI) } & $<0.001$ \\
\hline Fairly good & $5(3)$ & $57(26)$ & - \\
\hline Fairly bad & $18(10)$ & $80(36)$ & - \\
\hline Very bad & $163(87)$ & $83(38)$ & - \\
\hline \multicolumn{4}{|l|}{ Prognostic indicators } \\
\hline $\begin{array}{l}\text { Duration of } \\
\text { ICU stay }\end{array}$ & $5.5 \pm 5.4$ & $3.0 \pm 2.5$ & $<0.001$ \\
\hline $\begin{array}{l}\text { Duration of } \\
\text { hospital stay }\end{array}$ & $20.5 \pm 11.9$ & $19.5 \pm 17.2$ & 0.504 \\
\hline 28-day mortality & $25(13)$ & $9(4)$ & 0.001 \\
\hline \multicolumn{4}{|l|}{ Biochemical indicators } \\
\hline $\mathrm{PH}$ & $7.37 \pm 0.10$ & $7.40 \pm 0.10$ & 0.422 \\
\hline $\mathrm{PaO}_{2}$ & $128.6 \pm 63.2$ & $135.1 \pm 58.1$ & 0.284 \\
\hline $\mathrm{PaCO}_{2}$ & $128.6 \pm 63.3$ & $39.3 \pm 14.3$ & 0.787 \\
\hline $\mathrm{Lac}^{2}$ & $3.3 \pm 8.5$ & $2.1 \pm 2.7$ & 0.052 \\
\hline WBC & $14.8 \pm 6.9$ & $14.2 \pm 7.3$ & 0.352 \\
\hline $\mathrm{Hb}$ & $96.0 \pm 24.3$ & $98.5 \pm 23.1$ & 0.285 \\
\hline PLT & $146.5 \pm 73.2$ & $162.7 \pm 87.9$ & 0.047 \\
\hline HCT & $29.5 \pm 7.1$ & $30.6 \pm 11.3$ & 0.234 \\
\hline PCT & $2.0[0.2,12.1]$ & $0.9[0.2,4.7]$ & 0.023 \\
\hline ALB & $28.5 \pm 5.9$ & $29.6 \pm 6.4$ & 0.082 \\
\hline TBIL, median (IQR) & $15.3[9.3,31.2]$ & $15.9[10.3,24.3]$ & 0.674 \\
\hline DBIL, median (IQR) & $7.2[4.1,14.9]$ & $7.4[4.45,11.15]$ & 0.503 \\
\hline ALT, median (IQR) & $22.9[13.5,49]$ & $21.0[13.0,39.4]$ & 0.155 \\
\hline AST, median (IQR) & $40.0[24.0,98.0]$ & $38.6[20.7,78.3]$ & 0.091 \\
\hline BUN, median (IQR) & $8.5[5.4,12.7]$ & $6.7[4.3,10.2]$ & $<0.001$ \\
\hline Cr, median (IQR) & $110.0[83.0,165.0]$ & $92.0[72.0,123.5]$ & 0.027 \\
\hline $\mathrm{UA}$, mean $(\mathrm{SD})$ & $318.6 \pm 146.4$ & $297.6 \pm 142.5$ & 0.146 \\
\hline
\end{tabular}

aSD, standard deviation; ${ }^{\mathrm{b}} \mathrm{APACHE}$, acute physiology, age and chronic health evaluation; 'SOFA, sequential organ failure assessment; MODS, multiple organ dysfunction syndrome.

$p=0.034[0.010-0.829])$, and an older age $(\mathrm{OR}=1.100, p=0.022$ [1.014-1.194]) were found to be the risk factors for disease. There were no notable risk factors of delirium in those with lung or cerebral disease, though the patient numbers were low in these groups (Table 2). 


\subsection{Prognosis of Delirium in Different Diseases Types}

The length of ICU stays and 4-week survival times were compared through multivariate stepwise Cox regression models in delirium and non-delirium groups. We found that in all patient groups, delirium led to a significantly longer ICU stay $(p<0.001)$ and higher 28-day mortality $(p=0.001)$.

The length of ICU stays in those with cardiac disease $(p<0.001)$, pulmonary disorders $(p=0.011)$, septic shock $(p<0.001)$, cerebral

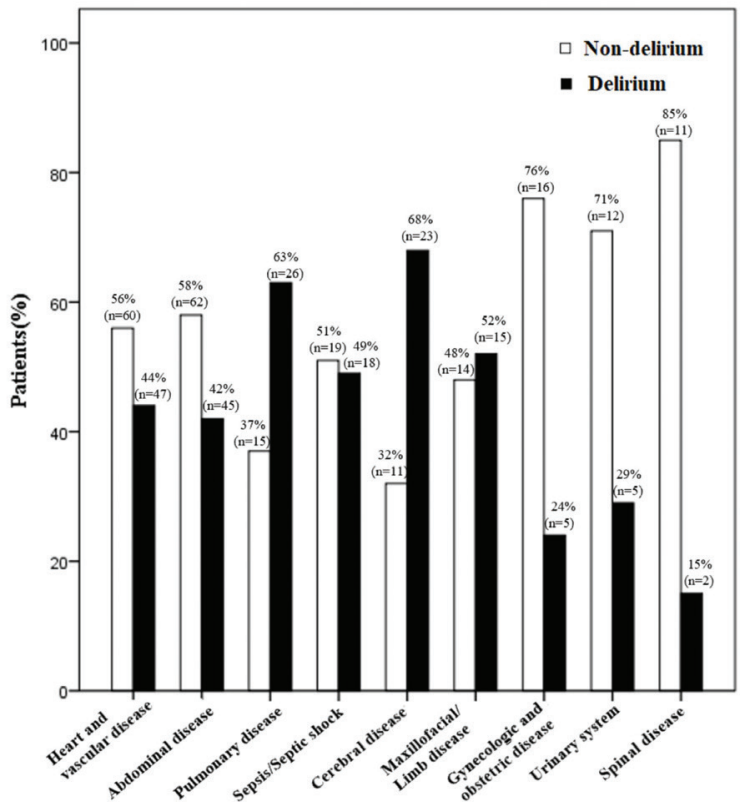

Figure 2 Delirium according to disease types. The symptoms of delirium were assessed twice daily by two ICU clinicians. Delirium was defined as: (1) acute changes or fluctuations in mental state; (2) loss of attention; (3) changes in consciousness; and (4) unordered thoughts. Delirium was considered present at values of: $1+2+3$ or $1+2+4$. Patients with delirium were positive when presenting at least one positive CAM-ICU during the ICU stay. In total, 186/406 patients had delirium. disease $(p=0.011)$ and intestinal disease $(p=0.47)$ were comparable to the general population (Figure 3 ). Only the subgroup of septic shock patients showed higher 28-day mortality in comparison to other patients with delirium ( $p=0.006$, Figure 4$)$.

\section{DISCUSSION}

Given the severe condition of ICU patients, delirium is common, with incidence rates ranging from $11 \%$ to $80 \%$, which can lead to prolonged ICU stays, mortality, cognitive decline, and an increased risk of death after 6 months [1-3]. In our study, of the 406 patients assessed, 186 (45.8\%) had delirium in the ICU. We demonstrated that delirium is strongly associated with a poor prognosis that varies according to the underlying disease types. A range of independent risk factors were identified to be associated with delirium upon the analysis of the disease characteristics of each patient subgroup. We found that ICU delirium was related to a poor prognosis with longer ICU stays and higher 28-day mortality. Amongst them, those with septic-shock associated delirium had the highest 28-day mortality.

For this study the symptoms of delirium were assessed twice daily by two ICU clinicians using the CAM-ICU scales. We found that delirium occurred in $45.8 \%$ of patients (range: $15-68 \%$ ) which was comparable to previous assessments in those with sepsis $[6,18]$. In a Japanese study, $27.8 \%$ of patients developed delirium in the ICU. In cardiac patients, delirium rates of $28 \%$ have been reported. Delirium was also shown to occur to high rates in transapical Transcatheter Aortic Valve Implantation (TAVI) (47\%) patients, but was less common in transfemoral TAVI (17\%) [10]. It probably depends on the type of disease, and our study included a variety of diseases that were common in the ICU, and the study showed that the overall incidence of delirium in the ICU was $45.8 \%$, suggesting that the incidence of delirium in the ICU was high.

We found that the use of sedatives, APACHE II scores, sleep disorders and ventilation duration could predict delirium. However, the risk factors varied according to disease types. The pathophysiological mechanisms of delirium development are largely undefined

Table 2 Multivariate regression analysis of the risk factors for delirium as a dependent variable in all patients and diseases types

Variables

All patients $(n=406)$

[p; $\left.\left(\mathrm{OR}^{\mathrm{a}}, 95 \% \mathrm{CI}\right)\right]$
Heart and vascular

disease $(n=104)$

[p; (OR, 95\% CI)]

\section{Abdominal disease \\ $(n=92)$ \\ [p; (OR, 95\% CI $)]$}

\section{Sepsis/Septic shock \\ $(n=37)$ \\ [p; (OR, 95\% CI)}

\begin{tabular}{|c|c|}
\hline $\begin{array}{c}\text { Cerebral } \\
\text { disease } \\
(n=31) \\
{[p ;(\mathrm{OR},} \\
95 \% \mathrm{CI})]\end{array}$ & $\begin{array}{c}\text { Pulmonar } \\
\text { disease } \\
(n=36) \\
{[p ;(\mathrm{OR},} \\
95 \% \mathrm{CI})]\end{array}$ \\
\hline
\end{tabular}

0.002 ;

$(2.514,1.397-4.524)$

0.022

$(1.100,1.014-1.194)$

$<0.001$

$(1.255,1.112-1.417)$

0.002 ;

$(13.799,2.669-71.361)$

$<0.001$;

$(0.114,0.036-0.366)$
0.034

$(0.09,0.010-0.829)$
(PSQI)

Duration of ventilation
$<0.001$;

$(0.186,0.121-0.288)$ 0.012 ;

(1.022,1.005-1.040)
(0.236, 0.111-0.500) 

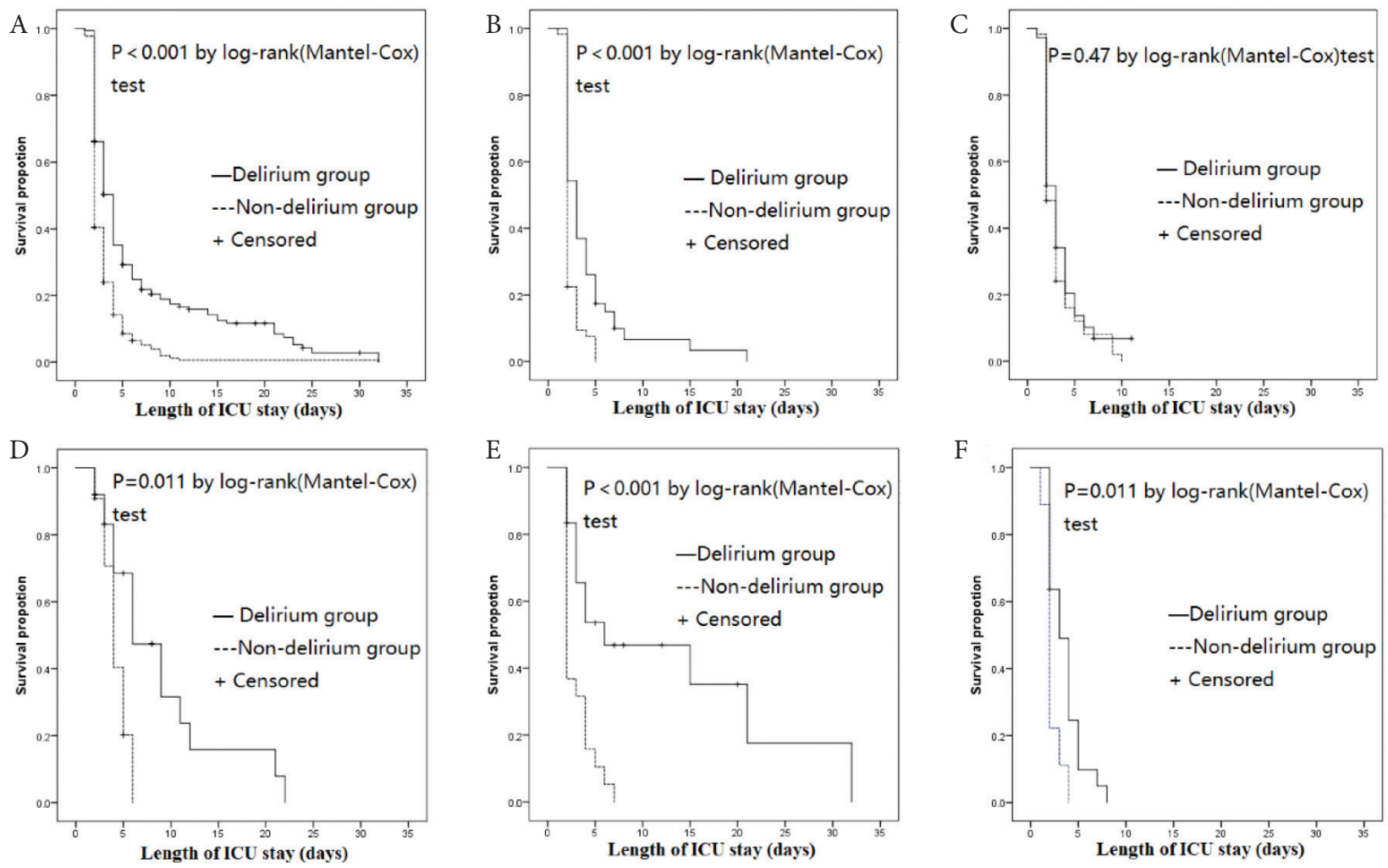

Figure 3 Length of ICU stay according to disease types in patients with or without delirium. Subgroups were classified according to disease types as described in materials and methods. Delirium was assessed as shown in Figure 1. A = Total patient cohort; B = Cardiac/vascular disease patients; $\mathrm{C}=$ Intestinal disorders patients; $\mathrm{D}=$ Pulmonary disorders patients; $\mathrm{E}=$ Septic shock patients; $\mathrm{F}=$ Cerebral disease patients.
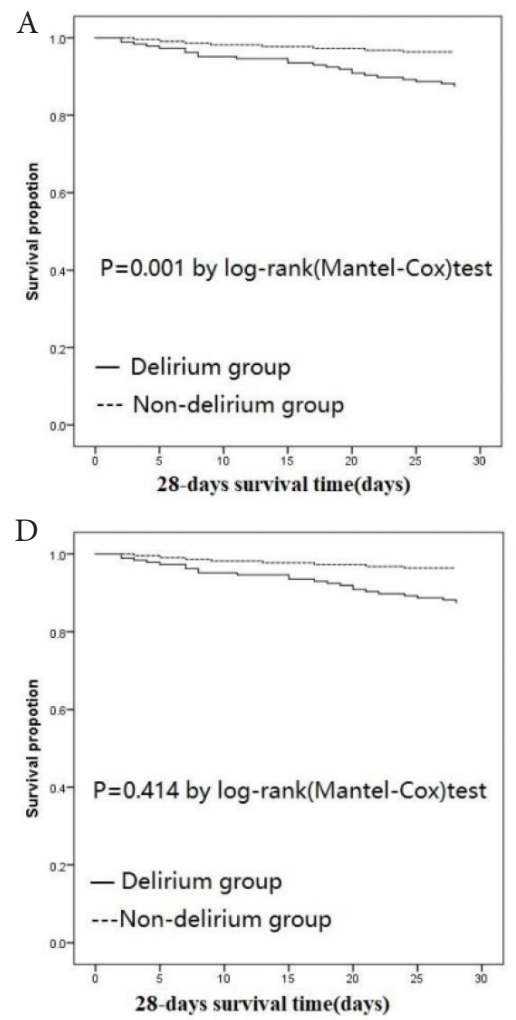
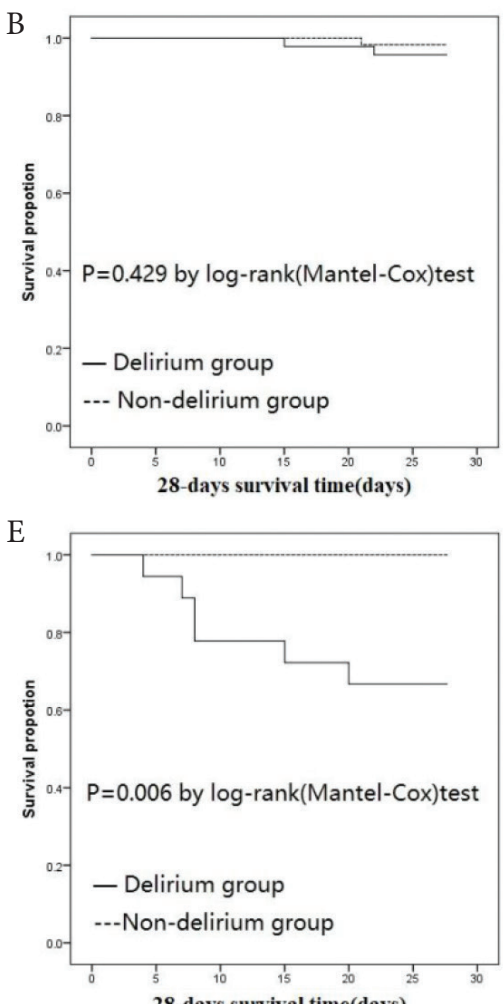

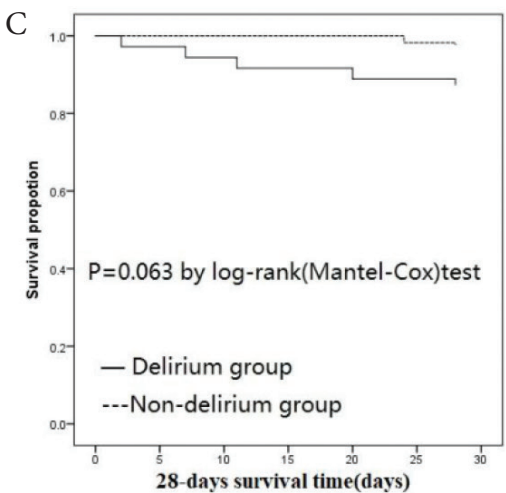

F

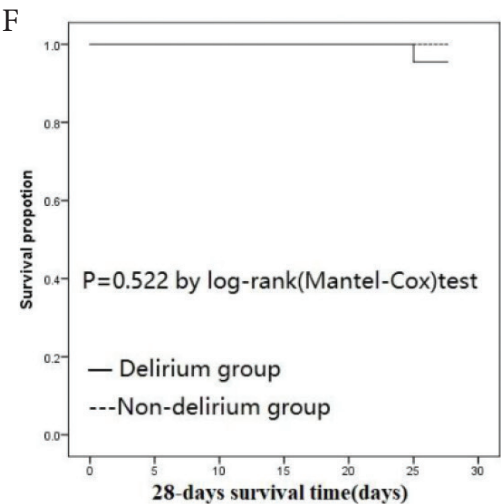

Figure 4 Rates of 28-day mortality between delirium and non-delirium groups. A = Total patient cohort; B = Cardiac/vascular disease patients; $\mathrm{C}=$ Intestinal disorders patients; $\mathrm{D}=$ Pulmonary disorders patients; $\mathrm{E}=$ Septic shock patients; $\mathrm{F}=$ Cerebral disease patients. 
[19] and are mainly dependent on the physiological characteristics of individual patients $[8,11,20]$. Yamaguchi et al. [9] found that older age and biopsychosocial vulnerability revealed through the COMPRI scale, led to a risk of ICU delirium and could therefore, predict mortality and longer ICU stays. Harten et al. [21] demonstrated that during cardiac surgery, the maintenance of a mean arterial pressure of $80-90 \mathrm{~mm} / \mathrm{Hg}$ reduced the rates of postoperative cognitive decline and delirium, suggesting that cerebral hypoperfusion/hypoxia contributes to changes in cognition. In our study, older age and lack of sleep were also identified as the risk factors for delirium. Previous studies [22-25] demonstrated that in unwell elderly individuals, the risk of delirium increased from $3 \%$ in those aged $\leq 65$ years to $14 \%$ in those aged $65-74$ years, with up to $36 \%$ of patients $\geq 75$ years $(p<0.0001)$. Older age was also a risk factor amongst hospitalized individuals, with Pandharipande et al. [24] showing a higher incremental risk in those aged $\geq 65$ years of transitioning to delirium by $2 \%$ for each year of life post-65 years (OR: 1.01, 95\% CI $=1.00,1.02, p=0.03$ ). These findings can be explained by a loss of neuronal function, changes in neurotransmitter systems, a loss of white matter integrity, a decreased regional cerebral blood flow, and a loss of neurochemical activity [26]. Besides, in patients with low sleep levels, delirium was frequent, suggesting the disruption of circadian rhythm as a possible cause for the onset [27-30]. Madrid-Navarro et al. [28] showed that exposure to continual noise, light and medication leads to poor sleep quality that ultimately causes delirium as a result of sleep disruption. The availability of drugs that can treat sleep disturbances in those who are critically ill is also sparse. Knauert et al. [29] suggested a range of multidisciplinary strategies to change the environment of the ICU and improve sleep and circadian rhythm, which may lead to a lower occurrence of delirium. It is therefore the responsibility of the healthcare professionals to provide evidence-based medicine in practice [31]. Prospective studies with larger sample sizes are needed to fully determine the specific risk factors for delirium [32,33].

Delirium is a complex disorder that is related to the severity of illness; environment; patient care; and iatrogenic factors. Considering the adverse impact of ICU delirium on critically ill patients, early identification is important to actively improve reversible risk factors. The effective participation of nurses and the treatment of underlying conditions that predispose patients to delirium can improve outcomes in patients receiving critical care [8]. There are no recommended preventive drugs or therapies for delirium. Non-drug interventions, including positive communication, early diagnosis in patients with psychological problems, family companionship, early mobilization therapeutic activities, optimization of hearing and vision, and improved sleep can improve patient support [34]. Patient outcomes may be improved by implementing preventive strategies for patients admitted to the ICU; early identification of the risk factors associated with delirium and taking positive corrective actions [35]. Despite the knowledge acquired to-date, everyday use of these therapies remains limited [36]. Delirium leads to poor long-term outcomes and high mortality rates as we observed that patients with delirium had longer times in the ICU and high rates of 28-day mortality. The prognosis of sepsis/shock patients with delirium was lower than those without, consistent with previous studies $[9,22]$. We previously showed comparable data [6] for patients with septic shock-associated delirium, in which more prolonged ICU stays and high mortality rates were observed. This may be related to the seriousness of the primary disease. In case of sepsis, long-term treatment is required to ameliorate the disease mediated neuronal cell damage, mitochondrial and endothelial dysfunction, a loss of neurotransmission, and altered neuronal $\mathrm{Ca}^{2+}$ homeostasis [37]. High mortality rates and long ICU stays have been reported as a result of extended resource consumption due to the high critical care burden [32]. Prolonged hospitalization can extend recovery times, increase the risk of patient falls, and heighten the risk of nosocomial infections amongst other undesirable effects [31]. Delirium experienced in the ICU can be long-lasting, with many survivors developing mental problems and subsequent cognitive impairment [17]. ICU patients with delirium struggle to separate fact from fiction and experience memory loss [19,38]. Attempts to understand the experience of ICU patients with delirium are therefore required to fully reveal its downstream consequences in surviving patients.

From our results, it was noteworthy that delirium did not influence the 28-day mortality for specific disease states. For example, the occurrence of delirium did not influence those with an abdominal disease. This may be explained by the fact that those with intestinal problems were treated with surgery and thus their disease was transient and rapidly treated, with no prolonged ICU stays required. This contrasted the findings of Raats et al. [39] who reported a regular occurrence of delirium in those undergoing abdominal surgery. It was further reported that delirium led to prolonged hospital stays, high mortality rates and the subsequent discharge to a nursing home. In contrast to other studies $[40,41]$, we observed changes in the duration of mechanical ventilation time due to delirium in those with pulmonary disease. This may be explained by the occurrence of respiratory failure, lack of treatment efficacy, and the low number of patients analyzed in this disease subgroup.

Our study protocol had some limitations. First, this was singlecenter observational study and the number of ICU patients with delirium that were analyzed was small. Second, we evaluated delirium twice per day and if this occurred outside of the assessment time, misdiagnosis could result. Third, the subgroup of patients with cerebral diseases included patients with hypertensive intracerebral hemorrhage, ruptured aneurysm hemorrhage, subarachnoid hemorrhage, brain injury and other newly emerging cerebral diseases without previous mental system illness and cognitive disorders. Thus, we cannot rule out that these emerging cerebral diseases may affect the cognitive function. Finally, we did not perform an analysis of the long-term prognosis. Futher studies may be carried out with specific focus on each disease type in the ICU in larger patient cohorts to determine the causality and etiology of the risk factors for delirium.

\section{CONCLUSION}

The incidence of delirium and its associated risk factors varied according to disease type. The risk factors for delirium were found to be disease specific. However, older age and poor sleep quality were identified as common risk factors in most disease subgroups. While ICU delirium led to poor outcomes, with longer ICU stays and higher 28-day mortality, the highest 28-day mortality was associated with septic-shock associated delirium. 


\section{CONFLICTS OF INTEREST}

The authors declare they have no conflicts of interest.

\section{AUTHORS' CONTRIBUTION}

Lina Zhang, Yuhang Ai, Meilin Ai, Li Huang, Qianyi Peng and Qing Feng contributed to the study conception and design. Lina Zhang, Yuhang Ai and Qianyi Peng performed the data analysis and interpretation. Qing Feng and Li Huang revised the manuscript for important intellectual content. Lina Zhang, Yuhang Ai, Li Huang, Qianyi Peng and Qing Feng gave final approval of the version to be published. All authors read and approved the final manuscript.

\section{FUNDING}

This work was supported by the National Natural Science Foundation of China: 81873956.

\section{ABBREVIATIONS}

CAM-ICU, confusion assessment method for the intensive care unit; CI, confidence interval; IQR, interquartile range; RASS, Richmond Agitation Sedation Scale; PSQI, Pittsburgh Sleep Quality Index; WBC, white blood cell; Hb, hemoglobin; PLT, platelet; HCT, hematocrit; PCT, procalcitonin; ALB, albumin; TBIL, total bilirubin; DBIL, direct bilirubin; ALT, glutamic-pyruvic transaminase; AST, glutamic-oxalacetic transaminase; BUN, blood urea nitrogen; $\mathrm{Cr}$, creatinine; UA, uric acid.

\section{ETHICAL APPROVAL AND CONSENT TO PARTICIPATE}

We ensure that the research described has been carried out in accordance with the Ethical Principles for Medical Research Involving Human Subjects (Declaration of Helsinki) of the World Medical Association. Central South University, Xiangya Hospital Ethics Committee approval the study, all patients involved in the study are based on the principle of voluntary, and have signed an informed consent form. We will maximize the protection of the interests of patients, and will not cause harm to all patients.

\section{REFERENCES}

[1] Ely EW, Shintani A, Truman B, Speroff T, Gordon SM, Harrell FE, et al. Delirium as a predictor of mortality in mechanically ventilated patients in the intensive care unit. JAMA 2004;291; 1753-62.

[2] Ramoo V, Abu H, Rai V, Surat Singh SK, Baharudin AA, Danaee M, et al. Educational intervention on delirium assessment using confusion assessment method-ICU (CAM-ICU) in a general intensive care unit. J Clin Nurs 2018;27;4028-39.

[3] Ely EW, Inouye SK, Bernard GR, Gordon S, Francis J, May L, et al. Delirium in mechanically ventilated patients: validity and reliability of the confusion assessment method for the intensive care unit (CAM-ICU). JAMA 2001;286;2703-10.

[4] Dessap AM, Roche-Campo F, Launay JM, Charles-Nelson A, Katsahian S, Brun-Buisson C, et al. Delirium and circadian rhythm of melatonin during weaning from mechanical ventilation: an ancillary study of a weaning trial. Chest 2015;148;1231-41.

[5] Souza-Dantas VC, Póvoa P, Bozza F, Soares M, Salluh J. Preventive strategies and potential therapeutic interventions for delirium in sepsis. Hosp Pract (1995) 2016;44;190-202.

[6] Feng Q, Ai YH, Gong H, Wu L, Ai ML, Deng SY, et al. Characterization of sepsis and sepsis-associated encephalopathy. J Intensive Care Med 2017;34;938-45.

[7] Zhang WY, Wu WL, Gu JJ, Sun Y, Ye XF, Qiu WJ, et al. Risk factors for postoperative delirium in patients after coronary artery bypass grafting: a prospective cohort study. J Crit Care 2015;30; 606-12.

[8] Mori S, Takeda JRT, Carrara FSA, Cohrs CR, Zanei SSV, Whitaker IY. Incidence and factors related to delirium in an intensive care unit. Rev Esc De Enferm USP 2016;50;587-93 [Article in English, Portuguese].

[9] Yamaguchi T, Tsukioka E, Kishi Y. Outcomes after delirium in a Japanese intensive care unit. Gen Hosp Psychiatry 2014;36;634-6.

[10] Tse L, Schwarz SKW, Bowering JB, Moore RL, Barr AM. Incidence of and risk factors for delirium after cardiac surgery at a quaternary care center: a retrospective cohort study. J Cardiothorac Vasc Anesth 2015;29;1472-9.

[11] Wang J, Li Z, Yu Y, Li B, Shao G, Wang Q. Risk factors contributing to postoperative delirium in geriatric patients postorthopedic surgery. Asia Pac Psychiatry 2015;7;375-82.

[12] Ely EW, Margolin R, Francis J, May L, Truman B, Dittus R, et al. Evaluation of delirium in critically ill patients: validation of the Confusion Assessment Method for the Intensive Care Unit (CAM-ICU). Crit Care Med 2001;29;1370-9.

[13] Bergeron N, Dubois MJ, Dumont M, Dial S, Skrobik Y. Intensive care delirium screening checklist: evaluation of a new screening tool. Intensive Care Med 2001;27;859-64.

[14] Barr J, Fraser GL, Puntillo K, Ely EW, Gélinas C, Dasta JF, et al. Clinical practice guidelines for the management of pain, agitation, and delirium in adult patients in the intensive care unit. Crit Care Med 2013;41;263-306.

[15] Gusmao-Flores D, Salluh JIF, Chalhub RÁ, Quarantini LC. The confusion assessment method for the intensive care unit (CAM-ICU) and intensive care delirium screening checklist (ICDSC) for the diagnosis of delirium: a systematic review and meta-analysis of clinical studies. Crit Care 2012;16;R115.

[16] Gusmao-Flores D, Salluh JIF, Dal-Pizzol F, Ritter C, Tomasi CD, de Lima MASD, et al. The validity and reliability of the Portuguese versions of three tools used to diagnose delirium in critically ill patients. Clinics (São Paulo) 2011;66;1917-22.

[17] Seymour CW, Liu VX, Iwashyna TJ, Brunkhorst FM, Rea TD, Scherag A, et al. Assessment of clinical criteria for sepsis: for the third international consensus definitions for sepsis and septic shock (Sepsis-3). JAMA 2016;315;762-74.

[18] Zhang LN, Wang XT, Ai YH, Guo QL, Huang L, Liu ZY, et al. Epidemiological features and risk factors of sepsis-associated encephalopathy in intensive care unit patients: 2008-2011. Chin Med J (Engl) 2012;125;828-31.

[19] Thorsteinsdóttir SA, Sveinsdóttir H, Snædal J. Delirium after open cardiac surgery: systematic review of prevalence, risk factors and consequences. Laeknabladid 2015;101;305-11 [Article in Icelandic]. 
[20] Williams ST. Pathophysiology of encephalopathy and delirium. J Clin Neurophysiol 2013;30;435-7.

[21] Harten AEV, Scheeren TWL, Absalom AR. A review of postoperative cognitive dysfunction and neuroinflammation associated with cardiac surgery and anaesthesia. Anaesthesia 2012;67;280-93.

[22] Pinho C, Cruz S, Santos A, Abelha FJ. Postoperative delirium: age and low functional reserve as independent risk factors. J Clin Anesth 2016;33;507-13.

[23] Pendlebury ST, Lovett NG, Smith SC, Dutta N, Bendon C, LloydLavery A, et al. Observational, longitudinal study of delirium in consecutive unselected acute medical admissions: age-specific rates and associated factors, mortality and re-admission. BMJ Open 2015;5;e007808.

[24] Pandharipande P, Shintani A, Peterson J, Pun BT, Wilkinson GR, Dittus RS, et al. Lorazepam is an independent risk factor for transitioning to delirium in intensive care unit patients. Anesthesiology 2006;104;21-6.

[25] Maldonado JR. Delirium pathophysiology: an updated hypothesis of the etiology of acute brain failure. Int J Geriatr Psychiatry 2018;33;1428-57.

[26] Maldonado JR. Neuropathogenesis of delirium: review of current etiologic theories and common pathways. Am J Geriatr Psychiatry 2013;21;1190-222.

[27] Maldonado JR. Pathoetiological model of delirium: a comprehensive understanding of the neurobiology of delirium and an evidence-based approach to prevention and treatment. Crit Care Clin 2008;24;789-856, ix.

[28] Madrid-Navarro CJ, Sanchez-Galvez R, Martinez-Nicolas A, Marina R, Garcia JA, Madrid JA, et al. Disruption of circadian rhythms and delirium, sleep impairment and sepsis in critically ill patients. Potential therapeutic implications for increased light-dark contrast and melatonin therapy in an ICU environment. Curr Pharm Des 2015;21;3453-68.

[29] Knauert MP, Haspel JA, Pisani MA. Sleep loss and circadian rhythm disruption in the intensive care unit. Clin Chest Med 2015;36;419-29.

[30] Weinhouse GL. Delirium and sleep disturbances in the intensive care unit: can we do better? Curr Opin Anaesthesiol 2014;27;403-8.
[31] Pauley E, Lishmanov A, Schumann S, Gala GJ, van Diepen S, Katz JN. Delirium is a robust predictor of morbidity and mortality among critically ill patients treated in the cardiac intensive care unit. Am Heart J 2015;170;79.e1-86.e1.

[32] Volland J, Fisher A, Drexler D. Delirium and dementia in the intensive care unit: increasing awareness for decreasing risk, improving outcomes, and family engagement. Dimen Crit Care Nurs 2015;34;259-64.

[33] Theologou S, Giakoumidakis K, Charitos C. Perioperative predictors of delirium and incidence factors in adult patients post cardiac surgery. Pragmat Obs Res 2018;9;11-19.

[34] Whitehorne K, Gaudine A, Meadus R, Solberg S. Lived experience of the intensive care unit for patients who experienced delirium. Am J Crit Care 2015;24;474-9.

[35] Visser L, Prent A, van der Laan MJ, van Leeuwen BL, Izaks GJ, Zeebregts CJ, et al. Predicting postoperative delirium after vascular surgical procedures. J Vasc Surg 2015;62;183-9.

[36] Collinsworth AW, Priest EL, Campbell CR, Vasilevskis EE, Masica AL. A review of multifaceted care approaches for the prevention and mitigation of delirium in intensive care units. J Intensive Care Med 2016;31;127-41.

[37] Beloborodova NB, Ostrova IV. Sepsis-associated encephalopathy (Review). Gen Reanimatol 2017;13;121-39 [Article in Russian].

[38] Page VJ, Kurth T. Delirium on the intensive care unit. BMJ 2014;349;g7265.

[39] Raats JW, Steunenberg SL, Crolla RMPH, Wijsman JHH, te Slaa A, van der Laan L. Postoperative delirium in elderly after elective and acute colorectal surgery: a prospective cohort study. Int J Surg 2015;18;216-19.

[40] Hsieh SJ, Soto GJ, Hope AA, Ponea A, Gong MN. The association between acute respiratory distress syndrome, delirium, and in-hospital mortality in intensive care unit patients. Am J Respir Crit Care Med 2015;191;71-8.

[41] Aliberti S, Bellelli G, Belotti M, Morandi A, Messinesi G, Annoni G, et al. Delirium symptoms during hospitalization predict long-term mortality in patients with severe pneumonia. Aging Clin Exp Res 2015;27;523-31. 